Article

\title{
Post-Mortem 7.0-Tesla Magnetic Resonance Imaging of the Hippocampus in Progressive Supranuclear Palsy with and without Cerebral Amyloid Angiopathy
}

\author{
Jacques De Reuck *, Florent Auger, Nicolas Durieux, Claude-Alain Maurage, \\ Vincent Deramecourt, Charlotte Cordonnier, Florence Pasquier, Didier Leys and Regis Bordet \\ Unitè 1171 “Degenerative and Vascular Cognitive Disorders”, Université de Lille 2, 2275 Lille, France; \\ florent.auger@univ-lille2.fr (F.A.); nicolas.durieux@univ-lille2.fr (N.D.); \\ claude-alain.maurage@chru-lille.fr (C.-A.M.); vincent.deramecourt@chru-lille.fr (V.D.); \\ charlotte.cordonnier@univ-lille2.fr (C.C.); florence.pasquier@univ-lille2.fr (F.P.); didier.leys@univ-lille2.fr (D.L.); \\ regis.bordet@univ-lille2.fr (R.B.) \\ * Correspondence: dereuck.j@gmail.com; Tel.: +32-(0)-447-652-167
}

Received: 30 October 2020; Accepted: 18 December 2020; Published: 21 December 2020

\begin{abstract}
Introduction and Purpose: Cerebral amyloid angiopathy (CAA) can be observed in patients with progressive supranuclear palsy (PSP), though to a lesser degree than in Alzheimer's disease. The present post-mortem 7.0-tesla magnetic resonance imaging (MRI) evaluates whether CAA has an influence on the degree of hippocampal atrophy (HA) and on the incidence of associated micro-infarcts (HMIs) and cortical micro-bleeds (HMBs). Material and Methods: Eight brains with PSP-CAA were compared to 20 PSP brains without CAA. In addition to the neuropathological examination, the hippocampus was evaluated on the most representative coronal section with $\mathrm{T} 2$ and $\mathrm{T} 2{ }^{*}$-weighted MRI sequences. The average degree of HA was determined in both groups. The incidence of HMIs and HMBs was also compared as well as the frequency of cortical micro-infarcts (CoMIs) and cortical micro-bleeds (CoMBs) in the hemispheric neocortex. Results: The neuropathological examination showed a higher incidence of lacunar infarcts in the PSP-CAA brains compared to the PSP ones. With magnetic resonance imaging (MRI), the severity of HA and the incidence of HMIs and HMBs was similar between both groups. Additionally, the frequency of CoMIs and CoMBs in the neocortex was comparable. Conclusions: The association of CAA in PSP brains has no influence on the degree of HA and on the incidence of the small cerebrovascular lesions in the hippocampus as well as in the neocortex.
\end{abstract}

Keywords: neuropathology; magnetic resonance imaging; progressive supranuclear palsy; cerebral amyloid angiopathy; hippocampus; cerebral neocortex

\section{Introduction}

Progressive supranuclear palsy (PSP) is a sporadic disorder with tau pathology [1]. The isoforms of four-repeat tau due to splicing of exon 10 define the tau filantous aggregates [2]. Phosphorilated TDP-43 pathology can be observed in PSP, although some other studies suggest a lack of TDP-43 pathology [3].

There are only a few studies concerning the hippocampal involvement in the pathology of PSP. In one study with magnetic resonance imaging (MRI), bilateral atrophy of both hippocampi together with the severe involvement of the thalami, the pallidum, and the brainstem is described [4]. However a fluorodeoxyglucose (FDG) positron emission tomography study showed, in addition to a global decrease in several regions of the cerebral hemispheres, a relative metabolic increase in the hippocampus [5]. 
Cerebral amyloid angiopathy (CAA) is absent in the normal aging brain [6]. It can be associated to PSP, but does not increase the overall incidence of small cortical vascular lesions, with the exception of cortical superficial siderosis [7].

Hippocampal atrophy (HA) in PSP is much less severe than in Alzheimer's disease and frontotemporal lobar degeneration [8].

The present post-mortem 7.0-tesla MRI study investigates whether the presence of CAA in PSP brains influences the degree of HA and the incidence of hippocampal micro-infarcts (HMIs) and hippocampal micro-bleeds (HMBs). Additionally, the frequency of cortical micro-infarcts (CoMIs) and cortical micro-bleeds (CoMBs) in the cerebral neocortex is compared between PSP-CAA and PSP brains.

\section{Material and Methods}

The examined post-mortem brains consisted of 8 PSP-CAA ones and 20 PSP ones without CAA. A previously obtained informed consent of the patients or from the nearest family allowed an autopsy for diagnostic and scientific purposes. The brain tissue samples were acquired from the Lille Neuro-Bank of the Lille University, which is part of the "Centres des Resources Biologiques" and acts as an institutional review board.

The neuropathological examination of PSP and associated small cerebrovascular lesions was made according to a previously described standard procedure [9].

The clinical diagnosis of PSP was made according to criteria proposed in 2017 by the international Parkinson and Movement Society, and was classified as probable, possible, and suggestive [10,11]. However, neuropathological examination allows a definite diagnosis.

The presence of various degrees of CAA was confirmed according to the criteria of a consensus protocol, and they were graded from 0 to 3 after examining four cortical samples with $\beta$-amyloid staining [12]. Grades 1 up to 3 were retained for diagnosis in the DLB-CAA group.

The degree of HA was determined according to the Alzheimer disease (AD) classification of Scheltens in 4 grades $[13,14]$. Additionally, the incidence of HMIs and HMBs was evaluated as previously described for cortical hemispheric CoMIs and CoMBs [15].

A 7.0-tesla MRI Bruker BioSpin SA was used with an issuer-receiver cylinder coil of $72 \mathrm{~mm}$ inner diameter (Ettlingen, Germany), according to a previously described method [16]. Before the brain sampling, three to six coronal sections of a cerebral hemisphere were submitted to SPIN ECHO T2 and T2* MRI sequences. The hippocampus was evaluated on the most representative section.

Unvaried comparisons of unpaired groups were performed with the Fisher's exact test for categorical data. The non-parametric Mann-Whitney $U$ test was used to compare continuous variables. The significance level, two-tailed, was set at $\leq 0.01$ for significant and $\leq 0.001$ for highly significant. Values set at $\leq 0.05$ and $>0.01$ were considered as marginally significant.

\section{Results}

There was no difference between the average age at death of the PSP brains with and without CAA, with 77 (SD: 11) years in the former group and 74 (SD: 9) in the latter groups. Additionally, no significant differences in gender distribution were observed: 50\% males in the PSP-CAA group and $42 \%$ in the PSP group without CAA.

There was no difference between the average age at death of the PSP brains with and without CAA, with 77 (SD: 11) years in the former group and 74 (SD: 9) in the latter group. Additionally, no significant differences in gender distribution were observed: $50 \%$ males in the PSP-CAA group and $42 \%$ in the PSP group without CAA.

The neuropathological examination revealed no statistical differences in the intensity of white matter changes and the incidence of territorial infarcts, lobar haematomas, cortical micro-infarcts, and cortical micro-bleeds in the neocortex of the cerebral hemispheres. Only lacunar infarcts were statistically more frequent in the PSP-CAA brains (Table 1). 
Table 1. Comparison on neuropathological examination of the average incidence of the cerebrovascular lesions between brains with progressive supranuclear palsy and cerebral amyloid angiopathy (PSP-CAA) and those without cerebral amyloid angiopathy (PSP).

\begin{tabular}{cccc}
\hline Items & PSP-CAA & PSP & $p$-Value \\
\hline White matter changes & $1.3(0.5)$ & $0.8(0.9)$ & NS \\
Territorial infarcts & $0.3(0.5)$ & $0.2(0.4)$ & NS \\
Lacunar infarcts & $0.8(0.5)$ & $0.1(0.2)$ & $<0.01$ \\
Lobar haematomas & $0.0(0.0)$ & $0.1(0.2)$ & NS \\
Cortical micro-infarcts & $0.5(0.6)$ & $0.6(0.8)$ & NS \\
Cortical micro-bleeds & $1.3(0.5)$ & $1.2(0.9)$ & NS \\
\hline
\end{tabular}

On MRI examination, no differences in the degree of HA and in the incidence of HMIs and HMBS was observed between the PSP-CAA brains and the "pure" PSP brains (Figures 1 and 2). Additionally, no statistical differences in average frequency CoMIs and CoMBs were observed in the neocortex of either group (Table 2).

Table 2. Comparison of the severity of the hippocampal atrophy and the incidence of hippocampal micro-infarcts and micro-bleeds, and the cortical micro-infarcts and micro-bleeds in the neocortex between brains with progressive supranuclear palsy associated to cerebral amyloid angiopathy (PSP-CAA) and those without this association (PSP) on magnetic resonance imaging.

\begin{tabular}{|c|c|c|c|}
\hline Items & PSP-CAA & PSP & $p$-Value \\
\hline Hippocampal atrophy & $0.6(0.7)$ & $0.9(0.6)$ & NS \\
\hline Hippocampal micro-infarcts & $0.0(0.0)$ & $0.2(0.4)$ & NS \\
\hline Hippocampal micro-bleeds & $0.9(0.6)$ & $0.4(0.5)$ & NS \\
\hline Neocortical micro-infarcts & $0.4(0.7)$ & $0.4(0.6)$ & NS \\
\hline Neocortical micro-bleeds & $1.0(0.9)$ & $1.1(1.2)$ & NS \\
\hline
\end{tabular}

Figure 1. $\mathrm{T} 2$ and $\mathrm{T} 2 *$ magnetic resonance imaging of a coronal section of a cerebral hemisphere in progressive supranuclear palsy associated with cerebral amyloid angiopathy. Note the hippocampal atrophy and the dilated temporal horn. A hippocampal micro-bleed is present on the $\mathrm{T} 2$ * sequence (black arrow). A micro-infarct in the cerebral neocortex is present on the T2 sequence. 


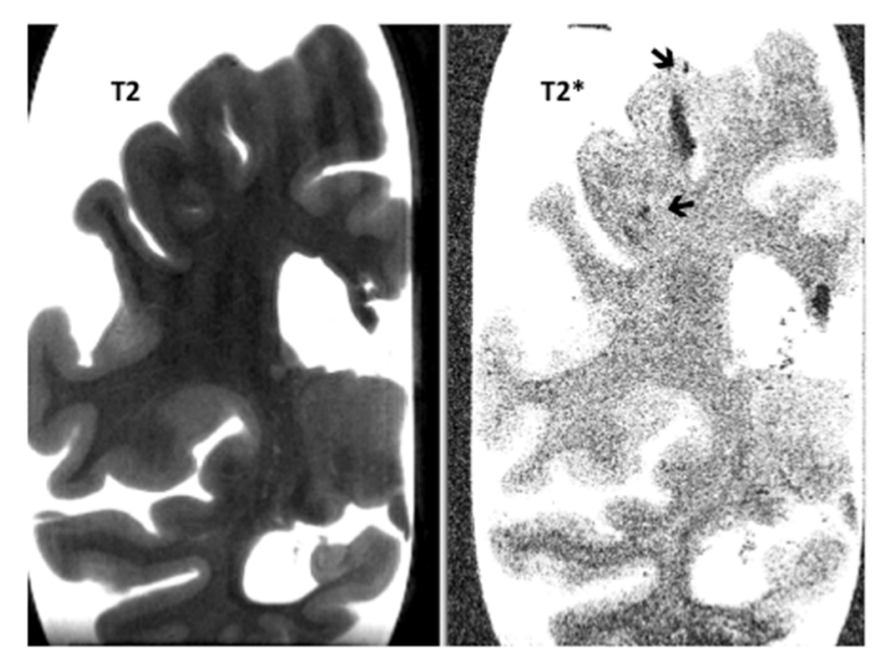

Figure 2. $\mathrm{T} 2$ and $\mathrm{T} 2 *$ magnetic resonance imaging of a coronal section of a cerebral hemisphere in progressive supranuclear palsy without cerebral amyloid angiopathy. Note the hippocampal atrophy and the dilated temporal horn. Some micro-bleeds are observed in the cerebral neocortex on the $\mathrm{T} 2{ }^{*}$ sequence (black arrows).

\section{Discussion}

7.0-tesla MRI allows a better detection of HMIs and HMBs than the 1.5 and 3.0 ones [17]. The present study shows that CAA has no influence on the degree of HA or on the incidence of the small hippocampal cerebrovascular lesions. These lesions are also comparable in the cerebral neocortex. On neuropathological examination, only lacunar infarcts were increased in the PSP-CAA group. No distinction could be made between them and dilated perivascular spaces [18].

PSP is possibly linked to corticobasal degeneration (CBD), and an overlap is not uncommon $[2,19]$. In our small series of five cases of $\mathrm{CBD}$, none had associated CAA. This observation does not allow definite conclusions on the relation between PSP and CBD. However, in both diseases, the HA is linked to TPD-43 pathology [3].

AOE E4 gene is absent in pure PSP brains [20] but can be present when an Alzheimer type of pathology is associated [21].

The severity of HA and the incidence of HMIs and HMBs is also not influenced by CAA in other neurodegenerative diseases such as AD disease [22] and in Lewy body dementia [23].

It therefore appears that the hippocampus in PSP brains is protected from additional damage caused by CAA. This is similar to the observations in other neurodegenerative diseases and in contrast to the high incidence of small cerebrovascular lesions in the cerebral neocortex [24].

Further studies are needed to clarify the significance of these findings.

Author Contributions: J.D.R. has designed the study. Together with F.A. and N.D. he performed the MRI examinations. C.-A.M. and V.D. performed the macroscopic and histological examinations of the brains. C.C., F.P., D.L. and R.B. were responsible for clinical evaluation during life. All authors have read and agreed to the published version of the manuscript.

Funding: No funding was received for the publication of this article.

Conflicts of Interest: The authors have nothing to declare in relation to this article.

\section{References}

1. Kovacs, G.G. Tauopathies. Handb. Clin. Neurol. 2017, 145, 355-368. [PubMed]

2. Scaravilli, T.; Tolosa, E.; Ferrer, I. Progressive supranuclear palsy and corticobasal degeneration: Lumping versus splitting. Mov. Disord. 2005, 20 (Suppl. S12), 21-28. [CrossRef] [PubMed] 
3. Yokota, O.; Davidson, Y.; Bigio, E.H.; Ishizu, H.; Terada, S.; Arai, T.; Hasegawa, M.; Akiyama, H.; Sikkink, S.; Pickering-Brown, S.M. Phosphorylated TDP-43 pathology and hippocampal sclerosis in progressive supranuclear palsy. Acta Neuropath. 2010, 120, 55-66. [CrossRef] [PubMed]

4. Saini, J.; Bagepally, B.S.; Sandhya, M.; Pasha, S.A.; Yadav, R.; Thennarasu, K.; Pall, P.K. Subcortical structures in progressive supranuclear palsy: Vertex-based analysis. Eur. J. Neurol. 2013, 20, 493-501. [CrossRef] [PubMed]

5. Ge, J.; Wu, J.; Peng, S.; Wu, P.; Wang, J.; Zhang, H.; Guan, Y.; Eidelberg, D.; Zuo, C.; Ma, Y. Reproducible network and regional topographies of abnormal glucose metabolism associated with progressive supranuclear palsy: Multivariate and univariate analyses in American and Chinese patient cohorts. Hum. Brain Mapp. 2018, 39, 2842-2858. [CrossRef]

6. De Reuck, J.; Auger, F.; Durieux, N.; Deramecourt, V.; Maurage, C.A.; Pasquier, F.; Cordonnier, C.; Leys, D.; Bordet, R. Cerebrovascular lesions during normal aging: A neuropathological study with 7.0-Tesla magnetic resonance imaging. EC Neurol. 2018, 10, 229-235.

7. De Reuck, J.; Auger, F.; Durieux, N.; Maurage, C.A.; Deramecourt, V.; Cordonnier, C.; Pasquier, F.; Leys, D.; Bordet, R. The impact of cerebral amyloid angiopathy in progressive supranuclear palsy: A neuropathological study with magnetic resonance imaging correlations. EC Neurol. 2019, 11, 807-812.

8. De Reuck, J.; Auger, F.; Durieux, N.; Maurage, C.A.; Deramecourt, V.; Cordonnier, C.; Pasquier, F.; Leys, D.; Bordet, R. Post-mortem 7.0-tesla magnetic resonance imaging of the hippocampus during normal aging and in neurodegenerative dementias. SunText Rev. Neurosci. Psychol. 2020, 1, 108.

9. De Reuck, J.; Caparros-Lefebvre, D.; Deramecourt, V.; Defebvre, L.; Auger, F.; Durieux, N.; Bordet, R.; Pasquier, F.; Maurage, C.-A. Prevalence of small cerebral bleeds in patients with progressive supranuclear palsy: A neuropathological study with 7.0-Tesla magnetic resonance imaging correlates. Folia Neuropathol. 2014, 52, 421-427. [CrossRef]

10. Höglinger, G.U.; Respondek, G.; Stamelou, M.; Kurz, C.; Josephs, K.A.; Lang, A.E.; Mollenhauer, B.; Müller, U.; Nilsson, C.; Whitwell, J.L.; et al. Clinical diagnosis of progressive supranuclear palsy: The movement disorder society criteria. Mov. Disord. 2017, 32, 853-864. [CrossRef]

11. Ali, F.; Martin, P.R.; Botha, H.; Ahlskog, J.E.; Bower, J.H.; Masumoto, J.Y.; Maraganore, D.; Hassan, A.; Eggers, S.; Boeve, B.F.; et al. Sensitivity and specificity of diagnostic criteria for progressive supranuclear palsy. Mov. Disord. 2019, 34, 1144-1153. [CrossRef] [PubMed]

12. Love, S.; Chalmers, K.; Ince, P.; Esiri, M.; Attems, J.; Jellinger, K.; Yamada, M.; McCarron, M.; Minett, T.; Matthews, F.; et al. Development, appraisal, validation and implementation of a consensus protocol for assessment of cerebral amyloidal angiopathy in post-mortem brain tissue. Am. J. Neurodegener. Dis. 2014, 3, 19-32. [PubMed]

13. Scheltens, P.; Leys, D.; Barkhof, F.; Huglo, D.; Weinstein, H.C.; Vermersch, P.; Kuiper, M.; Steinling, M.; Wolters, E.C.; Valk, J. Atrophy of medial temporal lobes on MRI in "probable" Alzheimer's disease and normal aging: Diagnostic value and neuropsychological correlates. J. Neurol. Neurosurg. Psychiatry 1992, 55, 967-972. [CrossRef] [PubMed]

14. Wahlund, L.O.; Julin, P.; Johansson, S.E.; Scheltens, P. Visual rating and volumetry of the medial temporal lobe on magnetic resonance imaging: A comparative study. J. Neurol. Neurosurg. Psychiatry 2000, 69, 630-635. [CrossRef]

15. De Reuck, J. Histopathological stainings and definitions of vascular disruptions in the elderly brain. Exp. Gerontol. 2012, 47, 834-837. [CrossRef]

16. De Reuck, J.; Auger, F.; Cordonnier, C.; Deramecourt, V.; Durieux, N.; Pasquier, F.; Bordet, R.; Maurage, C.; Leys, D. Comparison of 7.0-T T2*-magnetic resonance imaging of cerebral bleeds in post-mortem brain sections of Alzheimer patients with their neuropathological correlates. Cerebrovasc. Dis. 2011, 31, 511-517. [CrossRef]

17. Rotte, A.A.J.D.; Koning, W.; Hartog, A.G.D.; Bovens, S.M.; Zwanenburg, J.J.M.; Klomp, D.W.J.; Pasterkamp, G.; Moll, F.L.; Luijten, P.R.; De Borst, G.J.; et al. 7.0 t MRI detection of cerebral microinfarcts in patients with symptomatic high-grade carotid artery stenosis. Br. J. Pharmacol. 2014, 34, 1715-1719. [CrossRef]

18. Smith, E.E.; Schneider, J.A.; Wardlaw, J.M.; Greenberg, S.M. Cerebral microinfarcts: The invisible lesions. Lancet Neurol. 2012, 11, 272-282. [CrossRef]

19. Koga, S.; Kouri, N.; Walton, R.L.; Ebbert, M.T.W.; Josephs, K.A.; Litvan, I.; Graff-Radford, N.; Ahlskog, J.E.; Uitti, R.J.; Van Gerpen, J.A.; et al. Corticobasal degeneration with TDP-43 pathology presenting with progressive supranuclear palsy syndrome: A distinct clinicopathologic subtype. Acta Neuropathol. 2018, 136, 389-404. [CrossRef] 
20. Sabir, M.S.; Blauwendraat, C.; Ahmed, S.; Serrano, G.E.; Beach, T.G.; Perkins, M.; Rice, A.C.; Masliah, E.; Morris, C.M.; Pihlstrøm, L.; et al. Assessment of APOE in atypical parkinsonism syndromes. Neurobiol. Dis. 2019, 127, 142-146. [CrossRef] [PubMed]

21. Tsuboi, Y.; Jozephs, K.A.; Cookson, N.; Dickson, D.W. APAE E4 is a determinant for Alzheimer type of pathology in progressive supranuclear palsy. Neurology 2003, 60, 240-245. [CrossRef]

22. De Reuck, J.; Auger, F.; Cordonnier, C.; Deramecourt, V.; Durieux, N.; Pasquier, F.; Bordet, R.; Maurage, C.A.; Leys, D. Comparison of post-mortem 7.0-tesla magnetic resonance imaging of the hippocampus in Alzheimer brains with and without cerebral amyloid angiopathy. OBM Geriatr. 2020, in press.

23. De Reuck, J.; Auger, F.; Durieux, N.; Maurage, C.A.; Deramecourt, V.; Cordonnier, C.; Pasquier, F.; Leys, D.; Bordet, R. Comparison of post-mortem 7.0-tesla magnetic resonance imaging of the hippocampus in Lewy body dementia brains with and without cerebral amyloid angiopathy. EC Neurol. 2020, 12, 17-21.

24. De Reuck, J. The impact of cerebral amyloid angiopathy in various neurodegenerative dementia syndromes: A neuropathological study. Neurol. Res. Int. 2019, 2019, 7247325. [CrossRef] [PubMed]

Publisher's Note: MDPI stays neutral with regard to jurisdictional claims in published maps and institutional affiliations.

(C) 2020 by the authors. Licensee MDPI, Basel, Switzerland. This article is an open access article distributed under the terms and conditions of the Creative Commons Attribution (CC BY) license (http://creativecommons.org/licenses/by/4.0/). 